\title{
THE UPGRADE OF SRRC BOOSTER EXTRACTION SYSTEM
}

\author{
J. P. Chiou, J. S. Chen, C. S. Fann, C. H. Ho, K. T. Hsu, S. Y. Hsu, C. S. Hwang, \\ K. K. Lin", J. T. Sheu, T. S. Ueng, SRRC, Hsinchu, TAIWAN
}

\begin{abstract}
It is planned to upgrade the SRRC booster from $1.3 \mathrm{GeV}$ to $1.5 \mathrm{GeV}$ for full energy injection into the storage ring before the end of 1999. Based on the technical specification and operation experience, it is concluded that some of the subsystem components need to be rebuilt, such as the magnet power supply systems and beam extraction elements. A review of the power supply system will be reported elsewhere. In this report, the brief review of the beam extraction process is given according to the $1.5 \mathrm{GeV}$ parameters. The test result of a modified kicker magnet is presented.
\end{abstract}

\section{INTRODUCTION}

The SRRC booster had been operated as a full energy injector to the $1.3 \mathrm{GeV}$ storage ring since 1992 [1,2]. Beam energy ramping to the $1.5 \mathrm{GeV}$ in the storage ring was implemented in 1996 due to the increasing user's demand [3]. The limited capability of power supplies prevented booster from carrying out the $1.5 \mathrm{GeV}$ full energy injection. During the refilling of storage ring beam current, a routine process of energy ramping down (1.5 to $1.3 \mathrm{GeV}$ ), beam current accumulation, ramping up (1.3 to $1.5 \mathrm{GeV}$ ) was performed. The observed deficiency occurred with this process, in comparison with the full energy injection operating at $1.3 \mathrm{GeV}$, is described as follows. First, it takes typically 20 minutes for every refilling process. Second, the storage ring tunnel temperature was observed to become stable one hour after the completion of refilling process. It is due to the different power loading of components activated at 1.3 or $1.5 \mathrm{GeV}$. Third, it takes longer time for beam line elements to reach thermal equilibrium due to different refilling time in comparison with the case of full energy injection. Moreover, this accelerator configuration cannot provide the capability of performing top-up mode injection at $1.5 \mathrm{GeV}$. Consequently, a full energy injection at $1.5 \mathrm{GeV}$ is considered and is planned to implement in this year. Aside from the mentioned booster power supply limitation, the pulsed extraction system (bumpers, septum, and kicker) was also reviewed and decided to be modified. Fixtures of the septum coil need to be enforced in order to avoid insulation breakdown due to mechanical damage. Concerning kicker magnet, we modify this window frame ferrite, one-turn coil magnet into two-turn coil magnet, to reduce the amount of work and cost. The existing kicker power

\footnotetext{
*Email: kklin@srrc.gov.tw
}

supply is similar to that built in MAX [4] and will be preserved. In this report, we describe briefly the examination of extraction mechanism and the test result of the modified version of kicker magnet.

\section{EXTRACTION PROCESS}

\subsection{Description}

The SRRC booster is fast cycling at $10 \mathrm{~Hz}$. Electron beam injected at $50 \mathrm{MeV}$ is ramped up to $1.3 \mathrm{GeV}$ in $50 \mathrm{~ms}$ for extraction. As the accelerated beam approaches the extraction energy, three bumper magnets, with $2 \mathrm{~ms}$ half sine base width, are energized to produce a local orbit bump. This slow local orbit bump moves the beam orbit close to the extraction septum with largest amplitude at 1 ms, which corresponds to a duration of about 4000 turns after starting the extraction process. Then, the single turn extraction process is triggered by firing extraction kicker. Caution is made to properly trigger the extraction kicker so that the gap of bunch train, generated at the injection process, is utilized for the response duration of extraction kicker field. Thus, the extraction efficiency is optimized. When the kicker is triggered, an extra kick is added onto the horizontal deflection strength and deflects the electron beam to the other side of the septum magnet. The electron beam is then travelling along a 10 degrees bend trajectory and is injected into the transport line.

\subsection{Beam Parameters at Extraction of $1.5 \mathrm{GeV}$}

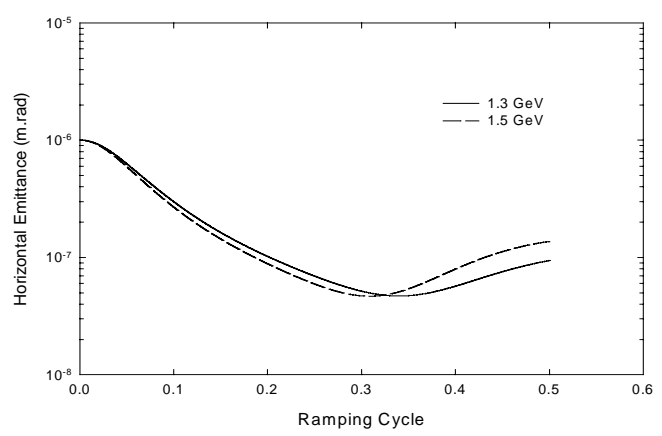

Figure 1: Calculated emittance variation of electron beam during ramping for both 1.3 and $1.5 \mathrm{GeV}$ cases.

As shown in figure 1, the calculated result shows a larger beam emittance at extraction for $1.5 \mathrm{GeV}$ than that for 1.3 $\mathrm{GeV}$. For the beam clearance consideration, the extraction bump amplitude at the septum location shall be adjusted 
accordingly. The cross sectional view of the stored, bumped, and kicked beam at the septum location in $x-p_{x}$ plane is depicted in figure 2 for illustration purpose.
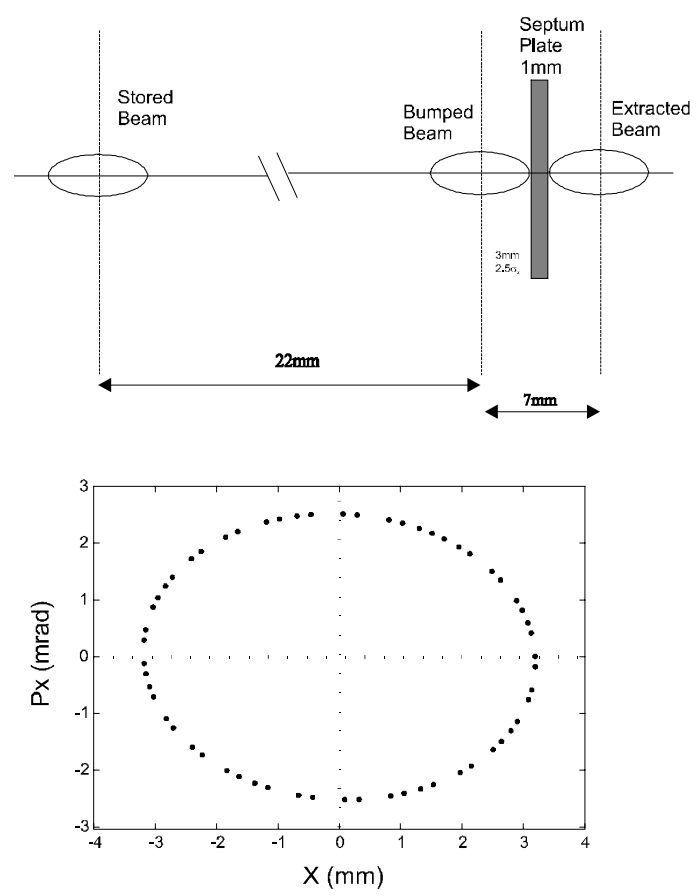

Figure 2: Tracking result on the $x-p_{x}$ plane of the extracted beam at $1.5 \mathrm{GeV}$. On top of the figure is the cross sectional view of the relative location of stored, bumped, and extracted beam at the septum entrance.

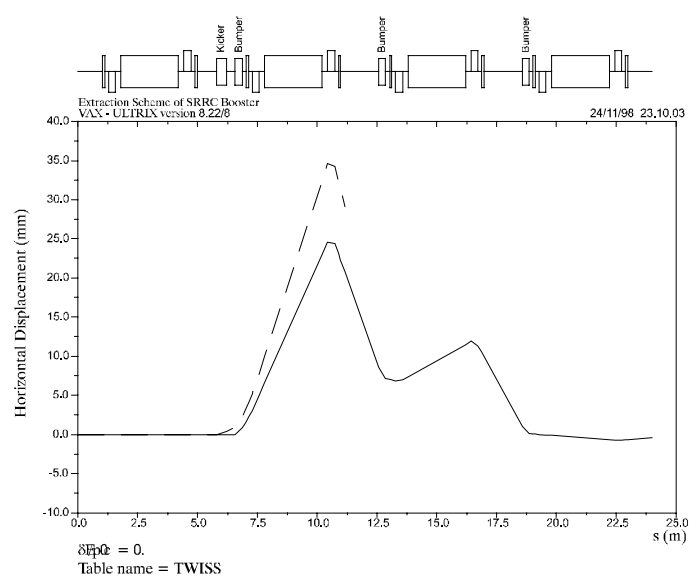

Figure 3: Trajectories of the electron beam centerline, bumped beam, and extracted beam after the ignition of extraction kicker. The figure shows two consecutive lattice cells in the extraction section.

Ellipses of tracking result shown in the figure cover the beam area of $2.5 \sigma_{x}$. In order to fulfill the criterion, the beam extraction process needs to be properly tuned. The calculated beam centerline, beam trajectory with local bump, and extracted beam trajectory, together with the schematic layout of the corresponding extraction elements at $1.5 \mathrm{GeV}$, are shown in figure 3 .
Considering the designed lattice for extraction at 1.5 $\mathrm{GeV}$, the kicker strength will require $30 \%$ increment over the present $1.3 \mathrm{GeV}$ case. This requirement is beyond the operating margin of the present unit. Therefore the extraction kicker system need to be modified. Among several possible options, it is chosen to modify kicker coil into two-turn unit in order to ease the amount of work.

\section{THE MODIFIED KICKER UNIT AND TEST RESULTS}

A modified two-turn coil with ferrite block was put into place instead of the present one-turn coil for testing purpose. The schematic layout of the testing unit is shown in figure 4 . Figure $4 \mathrm{a}$ and $4 \mathrm{~b}$ give the modified test unit of

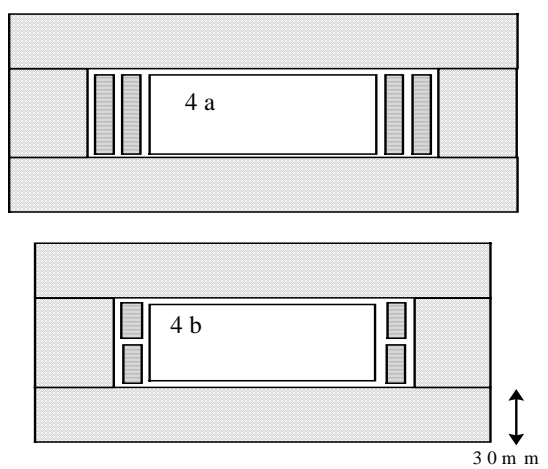

Figure 4: Window frame ferrite and coil arrangements of the (a) test unit of two-turn coil; and (b) two-turn coil of fabricating version.

different coil arrangement driven by the present power supply unit. The test unit of Fig. 4a type was assembled and tested in conjunction with daily operation at $1.3 \mathrm{GeV}$ for a short period of time. The result was encouraging. Figure 5 gives the comparison of the operation data between the original one-turn coil and the modified unit.

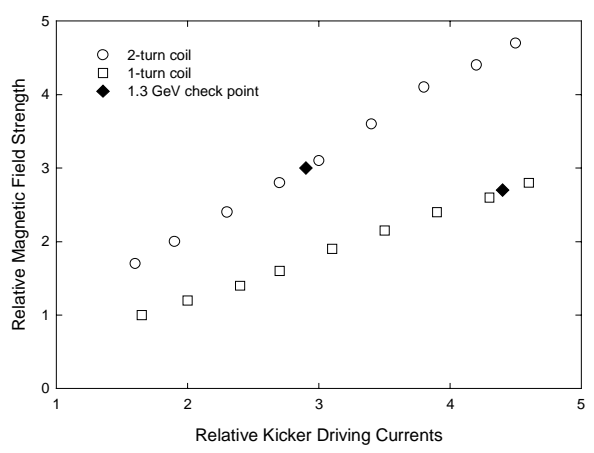

Figure 5: Comparison of the required kicker driving currents of one-turn coil and two-turns coil using the same existing power supply unit. Two check points working separately at daily operation of $1.3 \mathrm{GeV}$ electron beam extraction are also indicated in the figure. 
It shows that the driving current of the two-turn coil kicker could be largely reduced. According to the working points indicated in Fig. 5, this two-turn coil arrangement is capable of operation for $1.5 \mathrm{GeV}$ beam extraction.

In order to ease the engineering work and reduce the inductance of the kicker magnet, the two-turn coil was further modified and shown in Fig. 4b. The calculated result of Fig. $4 \mathrm{~b}$ arrangement is shown in figure 6 [5]. It shows that the field strength distribution and its horizontal uniformity is better than $0.3 \%$ over $+/-15 \mathrm{~mm}$ range which is larger than the region of interest. Therefore we have chosen the Fig. $4 \mathrm{~b}$ arrangement and it is under fabrication. The calculated result of field strength uniformity of Fig. $4 \mathrm{~b}$ is shown in figure 6.

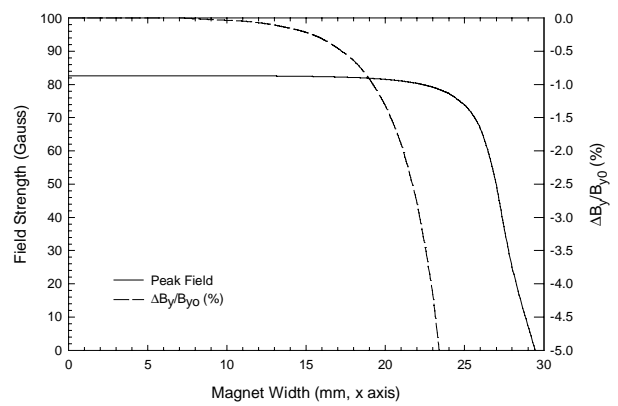

Figure 6: The kicker field strength in the region of interest is obtained using POISSON for two-dimensional calculation.

\section{SUMMARY}

In order to meet the requirement of operating SRRC booster at $1.5 \mathrm{GeV}$, engineering criterions for extraction process were reviewed in this report. The results suggest that the upgrade of SRRC extraction kicker is necessary. Among several alternatives, modifying one-turn coil into two-turn coil and preserving the existing power supply unit is chosen. The test result shows that the modified unit gives a satisfactory performance during daily operation at $1.3 \mathrm{GeV}$. This test unit also provides comfortable margin while operating at $1.5 \mathrm{GeV}$.

\section{REFERENCES}

[1] J. Modeer, "1.3 GeV electron synchrotron", Proceeding of PAC93, p.2034, Washington, D.C., May 17-20, 1993.

[2] K. K. Lin, K. T. Hsu, T. S. Ueng, J. P. Chiou, J. I. Hwang, Y. C. Liu, J. Modeer, "Performance of SRRC $1.3 \mathrm{GeV}$ electron booster synchrotron", Nucl. Inst. and Meth. in Phys. Res. A, 361(1995)1.

[3] G. H. Luo, P. Chang, K. T. Hsu, J. Chen, C. C. Kuo, Y. K. Lin, R. C. Sah, Y. C. Liu, "The $1.5 \mathrm{GeV}$ operation parameters and performance at SRRC”, Proceeding of PAC97, p.853.

[4] L. J. Lindgren, A. Sandell, M. Eriksson, "Fast kicker magnet system", Nucl. Instr. and Meth. 214(1983)179.

[5] POISSON/SUPERFISH reference manual, LA-UR-87-126, Los Alamos Accelerator Code Group, MS H829, LANL, Los Alamos, NM 87545, U. S. A. 